\title{
ELABORAÇÃO DE UM CURSO INTERATIVO VOLTADO AO APRENDIZADO DE UM SISTEMA APLICATIVO EM ODONTOLOGIA, UTILIZANDO A PLATAFORMA MOODLE
}

\section{CONSTRUCTION OF AN INTERACTIVE COURSE FOR LEARNING TO USE THE SOFTWARE FOR A DENTAL PRACTICE MANAGEMENT SYSTEM USING THE MOODLE PLATFORM}

\author{
Edivani Aparecida Vicente Dotta* \\ Patrícia Petromilli Nordi Sasso Garcia* \\ Lucas Miguel Candido***
}

\begin{abstract}
RESUMO
Introdução: Nas universidades, em qualquer tipo de faculdade ou instituto de pesquisa, é possível observar as inúmeras aplicações da informatização: gerenciamento de banco de dados, organização de catálogos, agilização de diagnósticos, entre outras. Dentro desse contexto, o ensino a distância pode facilitar esse aprendizado, porém os alunos, futuros prof ssionais, precisam estar preparados para lidar com as novas ferramentas disponibilizadas, usando-as em seu benefício e, consequentemente, em sua capacitação prof ssional, para a garantia de maior produtividade e interação com as diversas esferas no campo de trabalho. Este trabalho tem como objetivo desenvolver um curso a distância sobre a utilização de um sistema aplicativo odontológico. Métodos: Para isso, foi utilizada a plataforma Moodle, com seus recursos disponíveis. A elaboração do curso proposto foi dividida em duas partes: planejamento pedagógico e inserção do conteúdo pedagógico dentro dos recursos técnicos da plataforma Moodle. O curso foi composto por 14 módulos, com o intuito de possibilitar o aprendizado de forma progressiva e racional. Conclusôes: Concluiu-se que essa plataforma permitiu a elaboração do curso a distância proposto de forma prática e versátil, em função dos recursos e atividades que possui. Entretanto, para o uso dessa plataforma, deve-se ter domínio dos conceitos básicos de informática e das ferramentas da plataforma Moodle.
\end{abstract}

DESCRITORES: Informática odontológica • Odontológia • Ensino-aprendizagem • Educação a distância

\section{ABSTRACT}

Introduction: In universities, in any type of faculty or research institute, it is possible to observe innumerable informatics applications: database management, organization of catalogues, expediting diagnoses and many other applications. Within this context, distance learning could facilitate the learning of various professions. Distance education is the process of teaching-learning and the professional has to be prepared to deal with the new tools that are made available, using them to his/her benef $t$, and consequently in his/her professional capacity, to guarantee greater productivity and interaction with the various spheres in the work $\mathrm{f}$ eld. Methods: The aim of this research is to develop a course on the use of the Dentistry software application EasyDental, using the teaching at a distance methodology by means of the Moodle platform. The proposed course was composed of 14 modules, in order to enable a progressive and a rational learning. Conclusion: It could be concluded that this platform allowed the preparation of the proposed distance course, in a practical and versatile manner, considering the resources and activities it has. However, to use this platform, one must have dominion of the basic concepts of informatics, and the tools of the Moodle platform.

DESCRIPTORS: Dental informatics • Teaching-learning • Distance education

* Professor Assistente Doutor do Departamento de Odontologia Social, Faculdade de Odontologia de Araraquara, UNESP - Univ Estadual Paulista, Araraquara, SP, Brasil.

** Professor Adjunto do Departamento de Odontologia Social, Faculdade de Odontologia de Araraquara, UNESP - Univ Estadual Paulista, Araraquara, SP, Brasil.

*** Aluno de Graduação em Odontologia, Faculdade de Odontologia de Araraquara, UNESP - Univ Estadual Paulista, Araraquara, SP, Brasil. 


\section{N T R O D UÇ Ã O}

Considerando-se que o computador veio como uma ferramenta inovadora e facilitadora, sua utilização como instrumento de aprendizagem e sua ação no meio social é uma realidade, da qual não se pode fugir (Fidalgo-Neto et al. ${ }^{1}, 2009$, Kolikant $^{2}$, 2009), sendo, portanto, uma ferramenta que assume, cada vez mais, diversas funções no trabalho e no lazer (Rocha3 ${ }^{3}$ 2008).

Dessa forma, em uma sociedade informatizada como se transformou a nossa, o computador surgiu como ferramenta auxiliar na educação, se tornando um recurso promissor para a diminuição das carências. Petrin ${ }^{4}$ (2008), Mattei e Rausch ${ }^{5}$ (2008) também enfatizam que essa ferramenta pode potencializar mudanças no processo de ensino-aprendizagem com resultados promissores, possibilitando a criação de várias experiências de aprendizagem.

O papel da informática na educação, no tocante ao processo ensino-aprendizagem, sofreu muitas transformações ao longo dos anos (Carneiro ${ }^{6}, 2002$, Litto e Formiga $^{7}$, 2009) e, segundo Figueiredo e Bittencourt $^{8}$ (2005), ele faz parte de um processo natural do avanço da ciência. Há mais de 30 anos a informática é utilizada na educação; porém, apenas com o passar do tempo, começou a ser utilizada como uma ferramenta cognitiva, que num primeiro momento assumiu a função tradicional do professor como transmissor de conhecimento (Lévy9 , 1993).

Sales e Veiga ${ }^{10}$ (2009) salientam que o computador é um recurso auxiliar e facilitador do processo ensino-aprendizagem, favorecendo inclusive o desenvolvimento de habilidades cognitivas, assim como a autonomia dos alunos (Lévy9 ${ }^{9}$ 1993).

A internet é uma fonte de informações com grande penetração entre estudantes, possibilitando uma integração de conteúdos e contribuindo no desenvolvimento da autonomia, comunicação e inserção pessoal (Esteves Neto e Rocha11, 2009, Flores $^{12}$, 2000, Mendes e Marziale ${ }^{13}$, 2007, Silva $\left.{ }^{14}, 2006\right)$. A sua implantação permitiu que pesquisadores, de todo o mundo, vislumbrassem uma oportunidade ímpar de suporte a inovações no processo educacional, abrindo perspectivas para a obtenção de maior acesso à educação (Struchiner e Gianella ${ }^{15}$, 2005).

Conforme Tavares $^{16}$ (2007) salienta, essas tendências da educação sinalizam para alunos mais autônomos, maduros e dispostos a aprender. Contudo, os ambientes devem fornecer tecnologias e facilidades para a sua implementação, objetivando viabilizar o processo ensino-aprendizagem.

A metodologia da Educação a Distância - EaD nas universidades é empregada desde o século XX. O seu maior diferencial hoje é o gasto com o curso, que é menor, pois diminui o custo do transporte, $\mathrm{e}$ a sua interatividade que se tornou possível com a velocidade de transmissão via satélite (Bastos e Macedo ${ }^{17}, 2002$ ).

Vale reforçar que a EaD é uma modalidade de educação assim como a presencial (Nascimento e Carnielli ${ }^{18}, 2007$ ) e tem que apresentar boa qualidade, ou seja, deve auxiliar o aluno a aprender da mesma forma que a presencial, com seriedade e coerência no projeto pedagógico (Struchiner e Gianella ${ }^{15}, 2005$, Moran $^{19}$, 2000, Santos ${ }^{20}$, 2000).

Para a realização de um curso a distância é necessária a escolha de um sistema que esteja direcionado para o objetivo do curso. Esses sistemas são conhecidos como Ambientes Virtuais de Aprendizagem a Distância (AEaD) ou Plataforma de Ensino a Distância, entre outros, e são utilizados para autoria de cursos a distância por intermédio da internet (Silva $\left.{ }^{14}, 2006\right)$.

Esses ambientes virtuais permitem ao usuário, professor/tutor ou coordenador do curso, aplicar suas ideias e utilizar o computador sem a necessidade de possuir alguma habilidade em programação, porém com noções básicas de informática. Eles são destinados ao desenvolvimento de atividades on-line, envolvendo diversos aspectos tecnológicos, f nanceiros, administrativos e/ou pedagógicos (Alves e Brito $^{21}$, 2009), pois possuem recursos telemáticos (telecomunicação e informática) suf cientes para auxiliar na construção do conhecimento (Silva ${ }^{14}, 2006$ ), buscando um redimensionamento de conceitos já conhecidos e permitindo a busca e com-
DOTTA EAV

GARCIA PPNS

CANDIDO LM

ELABORAÇÃO

DE UM CURSO

INTERATIVO

VOLTADO AO

APRENDIZADO

DE UM SISTEMA

APLICATIVO EM

ODONTOLOGIA

UTILIZANDO

A PLATAFORMA

MOODLE

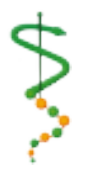

REV, ODONTOL.

UNIV, CID, SÃo

PAULO

2012; 24(1): 6-14, JAN - ABR 
DOTTA EAV :

GARCIA PPNS

CANDIDO LM

ELABORAÇÃO

DE UM CURSO

INTERATIVO

VOLTADO AO

APRENDIZADO

DE UM SISTEMA

APLICATIVO EM

ODONTOLOGIA

UTILIZANDO

A PLATAFORMA

MOODLE

8

REV. ODONTOL.

UNIV, CID, SÃO

PAULO

2012; 24(1): 6-14,

$J A N-A B R$
ISSN 1983-5183

preensão de novas ideias e valores (Valente ${ }^{22}$, 1999).

Segundo Moran ${ }^{23}$ (2009), os principais ambientes de aprendizagem são o Moodle, o Blackboard e o TelEduc, e algumas instituições desenvolvem seu próprio ambiente de aprendizagem.

O ambiente de aprendizagem Moodle (Modular Object Oriented Dynamic Learning Environment) é gratuito e foi desenvolvido pelo australiano Martinn Dougiamas, em 1999, em sua pesquisa de doutoramento, tendo trabalhado anteriormente como administrador do ambiente WebCT (Lopes e Gomes ${ }^{24}$, 2007). Esse ambiente atualmente está disponibilizado em 82 idiomas, um deles o português, e é utilizado em 208 países, com um número cada vez maior de pessoas contribuindo para seu desenvolvimento (Sistemas de Formação Multimédia ${ }^{25}$, 2010).

O desenvolvimento de um curso interativo que possa ensinar os estudantes de Odontologia e também os cirurgiões-dentistas a utilizarem um programa aplicativo para a informatização do consultório odontológico é extremamente interessante e pertinente pois, de acordo com Dotta e Teles ${ }^{26}$ (2003), o computador é uma poderosa ferramenta de marketing que diferencia o cirurgião-dentista perante um mercado cada vez mais competitivo; portanto, a informatização do ambiente odontológico é muito importante. Segundo os autores, a utilização de um sistema aplicativo permite o completo gerenciamento do consultório odontológico, tornando-se uma ferramenta fácil e prática para a organização das tarefas clínicas e administrativas de consultórios ou clínicas odontológicas.

Portanto, neste trabalho, foi selecionado como conteúdo para desenvolvimento de um curso interativo um sistema aplicativo na área de Odontologia, o EasyDental, pelo fato de tal sistema ser um dos mais utilizados pelos cirurgiões-dentistas, como constatado por Dotta ${ }^{27}$ (2004).

Diante disso, pode-se notar que o desenvolvimento de um curso interativo utilizando um ambiente de ensino a distância, no caso o Moodle, que habilite o cirurgião-dentista a administrar melhor seu ambiente prof ssional com a utiliza- ção da tecnologia, é pertinente e muito importante.

Assim, o presente trabalho tem como objetivo a elaboração de um curso interativo utilizando a plataforma de ensino a distância, Moodle, apresentando como se deve utilizar um sistema aplicativo em Odontologia, no caso específ co, o EasyDental.

\section{MÉTODOS}

Para a realização deste trabalho foi utilizada a plataforma Moodle. Além dela, vários comandos básicos (copiar, salvar, etc) da informática e sistemas foram usados: MovieMaker e PowerPoint para a confecção de todos os f Imes mostrados no curso, e o Adobe Acrobat para armazenamento de todos os arquivos/textos com terminação .pdf inseridos no curso.

Para f ns didáticos, a elaboração deste curso foi dividida em duas partes: o planejamento pedagógico e a inserção do conteúdo pedagógico dentro dos recursos técnicos da plataforma Moodle.

\section{A - Planejamento Pedagógico}

Ao fazer o planejamento pedagógico desse curso a distância foi possível constatar o quanto ele se assemelha com o de um curso presencial, pois, de acordo com Azevedo e Bittencourt ${ }^{28}$ (2005), as etapas são comuns: a primeira refere-se à def nição dos objetivos do curso, dos conteúdos e dos participantes; a segunda, como serão apresentados esses conteúdos no que tange ao material pedagógico e a terceira, como será o processo de avaliação desses participantes.

Esse curso foi realizado com o objetivo de desenvolver um aprendizado sobre a utilização do sistema em Odontologia, EasyDental, utilizando a metodologia de ensino a distância, por meio da plataforma Moodle.

Com relação aos participantes, inicialmente foram def nidos os três tipos de utilizadores do curso a ser desenvolvido (Sistemas de Formação Multimédia ${ }^{25}$, 2010):

1 - Administradores: A seleção do administrador foi o primeiro passo para a construção do curso, pois esse utilizador tem como função a execução de todo o 
trabalho de administração da plataforma, a alteração do aspecto do site, a inscrição de diferentes tipos de utilizadores, a def nição de suas permissões, etc. Para isso, solicitou-se ao Analista de Sistema do Serviço Técnico de Informática (STI) da Faculdade de Odontologia de Araraquara UNESP (FOAr), especialista na plataforma Moodle para assumir essa função;

2 - Professores e/ou Tutores: Para esse item foi selecionado o pesquisador responsável por esta pesquisa, uma vez que ele é também responsável pelas disciplinas envolvidas e pode fazer qualquer coisa dentro do curso, incluindo alteração das atividades e avaliações dos alunos;

3 - Alunos: Podem ter acesso aos conteúdos dos cursos em que se encontram inscritos. Qualquer um que se inscreva no curso pode ter acesso a ele, porém, num primeiro momento, o curso em questão foi direcionado para ser utilizado dentro da disciplina de Informática em Odontologia II do Curso de Odontologia da FOAr - UNESP, ministrada no segundo semestre, pelo pesquisador coordenador da responsável. A título de revisão também podem acessar o curso os alunos do $5^{\circ}$ ano do curso de Odontologia da FOAr - UNESP, dentro da disciplina de Informática e Documentação, que também é de responsabilidade do pesquisador responsável. Portanto, os participantes são alunos da graduação da FOAr - UNESP.

Convém ressaltar que dos utilizadores apresentados somente o administrador e professor/tutor podem desenvolver o curso, pois a eles é aberto um botão que permite essa edição.

No que se refere à apresentação do curso com relação ao material pedagógico, este possui:

1 - Um módulo inicial para a apresentação do curso;

2 - Treze módulos intermediários para a apresentação do sistema EasyDental, cada um deles trazendo uma avaliação do aprendizado;

3 - Um módulo f nal (Módulo 14) referente à "Avaliação Geral do Curso", destinado para sugestões dos alunos sobre o curso, podendo ser utilizado para futuras alterações do mesmo objetivando sua meIhoria.
O período de acesso ao curso e as avaliações de cada módulo têm tempo determinado. O acesso para os módulos é liberado durante todo o período do curso, porém, para as avaliações de cada módulo, inclusive do módulo f nal, há um peníodo determinado para sua realização. Em cada módulo, os alunos recebem notas que variam de 0 (zero) a 10 (dez), sendo considerados aprovados aqueles que obtêm média igual ou superior a 5,0 (cinco), considerando-se os 13 módulos de apresentação do Sistema EasyDental.

\section{B - Inserção do Conteúdo Pedagógico na Plataforma Moodle \\ Após a def nição dos utilizadores do curso, iniciou-se sua conf guração den-} tro da plataforma Moodle, feita pelo administrador, onde foram inseridos: Nome do curso; Objetivo; Formato; Número de Tópicos; Data de início do curso; Data de início e f nal das inscrições; Formato para inscrições (plugins) e Idioma.

O conteúdo do material pedagógico foi def nido e inserido pelo professor/tutor e os recursos e atividades (ferramentas) da plataforma Moodle foram utilizados. Vale ressaltar que na plataforma Moodle são considerados recursos as ferramentas que possuem informações estáticas, ou seja, sem interação entre professor/aluno. Por outro lado, as atividades são ferramentas que possibilitam essa interação (Bernardino e Fernandes ${ }^{29}$, 2009).

Para facilitar o entendimento da criação do curso usando-se a plataforma Moodle, estabeleceu-se que os seus recursos, suas atividades e suas categorias estarão escritos entre $<>$ e os resultados dos mesmos na tela aparecerão entre aspas (" ").

Os recursos e atividades do Moodle utilizados foram:

Recursos:

$<$ Criar uma página de texto simples $>$ : Permite inserir e editar textos e f guras;

$<$ Inserir rótulo $>$ : Permite inserir e editar textos e f guras, porém não cria um link e sim uma informação estática na tela;

$<$ Link a um arquivo ou site $>$ : Permite inserir arquivos que podem ser textos, apresentações, f Imes, etc.

$<$ Criar uma página web $>$ : Permite inseir texto e f gura e alterar para linguagem
DOTTA EAV

GARCIA PPNS

CANDIDO LM

ELABORAÇÃO

DE UM CURSO

INTERATIVO

VOLTADO AO

APRENDIZADO

DE UM SISTEMA

APLICATIVO EM

ODONTOLOGIA

UTILIZANDO

A PLATAFORMA

MOODLE

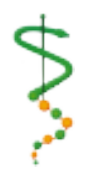

REV, ODONTOL,

UNIV, CID, SÃO

PAULO

2012; 24(1): 6-14, JAN - ABR 
DOTTA EAV :

GARCIA PPNS

CANDIDO LM

ELABORAÇÃO

DE UM CURSO

INTERATIVO

VOLTADO AO

APRENDIZADO

DE UM SISTEMA

APLICATIVO EM

ODONTOLOGIA

UTILIZANDO

A PLATAFORMA

MOODLE

\section{0}

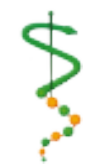

REV, ODONTOL.

UNIV, CID, SÃO

PAULO

2012; 24(1): 6-14,

$J A N-A B R$ da web (.html).

\section{Atividades:}

$<$ Fórum $>$ : Permite criar um espaço para comunicação entre os alunos e professores;

$<$ Questionário $>$ : Permite inserir as formas de avaliações. As categorias utilizadas neste curso foram: <Resposta breve $>$, $<$ Verdadeiro/Falso $>$, $<$ Associação $>$ e $<$ Múltipla Escolha $>$.

$<$ Pesquisa de avaliação $>$ : Permite que o aluno faça uma avaliação do curso e uma autoavaliação.

$<$ Escolha $>$ : Enquete onde o aluno atribuirá uma nota ao curso.

\section{DISCUSSÃO E RESULTADOS}

Após as fases de planejamento pedagógico e inserção do conteúdo pedagógico na plataforma Moodle, obteve-se o curso a distância.

O curso apresenta a seguinte conf guração:

Nome do curso: Curso interativo - Sistema aplicativo EasyDental

Objetivo: Desenvolver um aprendizado sobre a utilização do sistema EasyDental utilizando a plataforma Moodle.

Formato: Tópicos

Número de Tópicos: 14

Data do início do curso: Variável a cada ano

Inscrições

Plugins para inscrição: Padrão do site (Inscrição interna)

Data de início: A def nir

Data f nal: A def nir

Idioma: Português

O curso desenvolvido na modalidade à distância envolve a indicação de leituras, acompanhamento por meio de f Imes explicativos sobre a forma de utilização do conteúdo do sistema EasyDental e atividades de avaliação da aprendizagem do aluno, além da análise crítica feita pelo mesmo sobre o material didático desenvolvido. Dessa forma, neste curso tem-se:

Público Alvo: Alunos do $8^{\circ}$ semestre do curso de Odontologia da FOAr matriculados na disciplina de Informática em Odontologia II.

Carga Horária: Aproximadamente 15 horas, o que equivale a um crédito.
Período de Realização: Agosto a dezembro de cada ano

Local: Exceto um encontro presencial para explicação do funcionamento do curso, as demais atividades podem ser desenvolvidas no Laboratório Didático de Informática (LDI) da FOAr - UNESP ou nos locais mais apropriados para os alunos.

\section{Conteúdo}

Programação: Esse local é considerado uma área livre e foi destinado à apresentação do curso.

Para apresentação do Módulo inicial, utilizando-se o recurso $<$ Criar uma pasta de texto simples $>$, foram inseridos o logotipo do sistema EasyDental, criado pela empresa Easy Software S/A, bem como o nome e objetivo do curso. Com o recurso < Inserir rótulo $>$ foi possível inserir, à esquerda, o logotipo da versão 7.5 do sistema EasyDental. A atividade $<$ Fórum $>$ permitiu inserir dois links: o link "Instruções Didáticas" e o "Introdução", os quais foram desenvolvidos com o recurso $<$ Criar uma página web>.

Para inserir os fImes como links, "Apresentação de Sistemas Aplicativos e Ferramentas Automatizadas" e "Apresentação da abertura do sistema EasyDental" foi utilizado o recurso < link a um arquivo ou site>. Esses f Imes foram inicialmente criados e publicados no sistema Windows Movie Maker.

O item "Complementação de Estudos" foi criado por meio do recurso < Inserir rótulo $>$, e em função disso esse item não aparece como link e sim como organizador dessa área.

No link "Ambiente Virtual", foi inserido um artigo no formato .pdf, com o objetivo de complementar a leitura dos alunos e, para isso, utilizou-se o recurso $<$ link a um arquivo ou site>. Já no link "Capítulo de um livro", criado com o mesmo objetivo do anterior, utilizou-se o recurso $<$ Criar uma página na web $>$, pois é mostrada ao aluno somente a referência do capítulo indicado.

O conteúdo pedagógico que forma a maior parte do curso, dividido em 13 módulos, obedeceu à mesma estrutura. Em função disso, será explicado em conjunto. A estrutura padrão desses 13 módulos 
compõe-se de um texto de apresentação, uma f gura ilustrativa e três links padrões. O texto que aparece impresso na tela de cada módulo foi denominado de Texto de Apresentação dos Módulos, e para sua inserção utilizou-se o recurso da plataforma Moodle <criar uma pasta de texto simples>. A f gura ilustrativa, correspondente a cada módulo, foi inserida por meio do recurso < Inserir rótulo $>$. O aluno terá a oportunidade de aprender essas informações em dois momentos diferentes, nos seguintes links:

1 - "Material de estudo": Utilizando-se o recurso < link a um arquivo ou site> foi possível inserir um texto que permite ao aluno acompanhar o assunto relativo ao módulo por meio de um arquivo texto, no formato .pdf. Esse texto é oriundo do material entregue na compra of cial do EasyDental, isto é, refere-se ao Manual do sistema EasyDental.

2 - "Demonstração do módulo em questão": Utilizando-se o mesmo recurso da plataforma Moodle do item anterior, um f Ime demonstrativo, com as partes mais importantes de cada módulo, foi inserido. Esse f Ime foi desenvolvido por meio do sistema da Microsoft PowerPoint e do sistema Windows Movie Maker.

3 - "Atividades respectivas a cada módulo": Utilizando-se a atividade <Questionário > da plataforma Moodle foi possível aplicar diferentes formas de avaliação que são explicadas em cada atividade específ ca. Ao f nal de cada resposta dada pelo aluno, este é informado se ela está ou não dentro do esperado, por meio de uma frase escrita na tela.

A mudança dos paradigmas convencionais do ensino, através da introdução de novas mídias, poderá ser uma revolução na educação, à medida que evita que professores e alunos mantenham-se distantes. Se isso não ocorrer, dar-se-á apenas um verniz da modernidade, sem mexer no essencial (Moran $\left.{ }^{19}, 2000\right)$.

Nesse contexto, o computador pode ser um excelente auxiliar à medida que o professor e o aluno podem executar e elaborar atividades com auxílio da multimídia, em especial. Monteiro ${ }^{30}$ (2009) também af rma que o computador é uma grande promessa para uma nova revolu- ção no ensino.

Atualmente a EaD não é mais somente uma modalidade complementar de ensino e sim um eixo norteador de mudanças profundas da educação como um todo, principalmente no ensino superior (Moran $\left.{ }^{23}, 2009\right)$. Porém, não se deve esquecer que essa modalidade deve possuir qualidade igual ou superior à presencial, apresentando seriedade e coerência no seu projeto pedagógico como defendem Struchiner e Gianella ${ }^{15}$, 2005, Moran ${ }^{19}$, 2000 e Santos ${ }^{20}, 2000$ ).

O planejamento de um curso a distância é essencial para que ele apresente boa qualidade porque, segundo Lopes e Gomes $^{24}$ (2007), a falta de conteúdos adequados para alimentar os espaços a dinamizar, a adaptação de estratégias de ensino muito centradas no professor e nos conteúdos, a dinamização de atividades com interação e motivadoras para os alunos, entre outros, são aspectos muito importantes e que devem ser considerados no momento da elaboração do curso. Isso porque a tecnologia possibilita a utilização e dinamização dos espaços virtuais de aprendizagem, porém, se mal empregada, não ajudará numa construção adequada de um curso. Coscarelli ${ }^{31}$ (1998) também reforça essa ideia ao af rmar que a informática, assim como qualquer outra ferramenta que possa ser usada em situações de ensino-aprendizagem, depende do uso que se faz dela, não se podendo esperar milagres das novas tecnologias.

Dentro do planejamento de um curso de EaD, um aspecto a ser considerado é como ele será fundamentado. Segundo Silva $^{14}$, (2006), Alves e Brito ${ }^{21}$, (2009), Valente $^{22}$, (1999), a EaD é fundamentada na AEaD ou Plataforma de Ensino a Distância e esta, para ser completa, deve permitir, entre outras características, troca de comunicação entre os envolvidos no curso com ferramentas como e-mails, fóruns de discussão e salas de chats, reproduzindo não apenas um ambiente de sala de aula on-line.

Para a realização deste trabalho foram avaliados alguns ambientes de ensino a distância como o TelEduc, a WebCT e o Moodle, sendo este último o selecionado. A seleção ocorreu levando-se em conside-
DOTTA EAV

GARCIA PPNS

CANDIDO LM

ELABORAÇÃOO

DE UM CURSO

INTERATIVO

VOLTADO AO

APRENDIZADO

DE UM SISTEMA

APLICATIVO EM

ODONTOLOGIA

UTILIZANDO

A PLATAFORMA

MOODLE

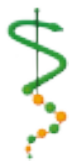

REV, ODONTOL.

UnIV. CID, SÃO

PAULO

2012; 24(1): 6-14, JAN - ABR 
DOTTA EAV

GARCIA PPNS

CANDIDO LM

ELABORAÇÃO

DE UM CURSO

INTERATIVO

VOLTADO AO

APRENDIZADO

DE UM SISTEMA

APLICATIVO EM

ODONTOLOGIA

UTILIZANDO

A PLATAFORMA

MOODLE

\section{2}

ração que o TelEduc, apesar de ser utilizado por várias instituições e ser gratuito, não possui uma estética de apresentação na tela e usabilidade que corresponda às expectativas dos usuários. Já a WebCT, apesar de ser uma plataforma que está sendo utilizada com resultados positivos em algumas faculdades, é paga (Bottentuit JR e Coutinho ${ }^{32}$, 2007). O Moodle, além de ser um ambiente de aprendizagem gratuito, com livre utilização de código aberto (open source), é projetado com princípios pedagógicos que auxiliam os educadores a criarem efetivamente cursos on-line, podendo formar comunidades virtuais de ensino. Esse ambiente é formado por um conjunto de quatro funções como o acesso protegido, ferramentas e serviços de comunicação síncrona e assíncrona, sistema de controle de atividades e disponibilização de conteúdos e de exercícios e avaliações. Essas funções foram essenciais para que o curso proposto pudesse ser realizado de forma fácil e funcional.

A interação e a dinâmica da plataforma Moodle também foram fatores primordiais para que Gonçalves e Salvador ${ }^{33}$ (2009) migrassem da plataforma WebCT para a Moodle. Além disso, a plataforma Moodle se destaca das demais por apresentar entre as suas principais características a adaptabilidade, ausência de custo na aquisição ou na obtenção de licenças e possibilidade de uso em qualquer sistema operacio- nal. Alves e Brito ${ }^{21}$ (2009) ressaltaram que essa plataforma possui amplos conceitos didáticos, contribuindo não somente para a EaD como também para o ensino presencial. As ferramentas de comunicação, de criação e de administração dos componentes de aprendizagem do Moodle, as possibilidades de poder ser instalado por qualquer usuário em todo o mundo, desde que tenha alguns conhecimentos mais específ cos em computação, também são características que muito contribuíram para execução adequada do curso apresentado.

Constatou-se que, ao se desenvolver cada etapa do curso, foi possível encontrar na plataforma Moodle a possibilidade de realizar de maneira livre e acessível seus recursos e atividades que permitiram a inserção de conteúdos educativos, promoção de atividades de aprendizagem e realização de avaliações dirigidas aos alunos e ao curso.

\section{CONCLUSÃO}

Pode-se concluir que a plataforma Moodle permitiu a elaboração do curso a distância proposto de forma prática e versátil em função dos recursos e atividades que possui. Entretanto, para o uso dessa plataforma, deve-se ter domínio dos conceitos básicos de informática e das ferramentas da plataforma Moodle. 
DOTTA EAV

GARCIA PPNS

CANDIDO LM

ELABORAÇÃO

DE UM CURSO

INTERATIVO

VOLTADO aO

APRENDIZADO

DE UM SISTEMA

APLICATIVO EM

odONTOLOGIA

UTILIZANDO

A PLATAFORMA

MOODLE

5. Mattei C, Rausch R. O prazer de aprender com a informática na educação infantil. 2008 [Acesso em 05 nov. 2008]; Disponível em: http://www.icpg.com.br/artigos/ rev02-11.pdf.

6. Carneiro R. Informática na educação: representação social do cotidiano: São Paulo; 2002.

7. Litto F, Formiga M. Educação à distância: o estado da arte. São Paulo: Pearson Education 2009.

8. Figueiredo C, Bittencourt J. Jogos computadorizados para aprendizagem matemática no ensino fundamental: ref etindo a partir dos interesses dos educandos. RENOTE Rev Novas Tecnol Educ 2005 3(1):1-10.

9. Lévy P. A metáfora do hipertexto. In: Lévy P. A tecnologia da inteligência. Rio de Janeiro: 34; 1993.

10. Sales I, Veiga R. O uso da informática no processo ensino-aprendizagem, estudo de caso na Universidade Vale do Rio Verde de Três Corações. 2009 [Acesso em 26 out. 2009]; Disponível em: http://www.unincor.br/Ousodainformatica.html.

11. Esteves Neto $\mathrm{H}$, Rocha S. A informática como ferramenta para a melhoria do processo ensino-aprendizagem na escola pública. 2009 [Acesso em 26 nov. 2009]; Disponível em: http://www.ie.ufmt.br/semiedu2006/CT10-Formaçãodeprofessores.

12. Flores S. Ensino a distância: paradoxos e aspectos psicológicos Psique (Belo Horizonte) 2000 maio 10(16):42-55.

13. Mendes IAC, Marziale MHP. Distance education: getting further. Rev Latino-Am Enfermagem 2007 Nov./Dec.;15(6):1059-60.

14. Silva J. Educação inclusiva em cursos de licenciatura: um estudo sobre possibilidades e limitações da educação a distância $(\mathrm{EaD})$ para formação de professores [Dissertação]. Araraquara: Faculdade de Ciências e Letras da UNESP; 2006.

15. Struchiner M, Gianella T. Educação a distância. In: Struchiner M, Gianella T. Aprendizagem e prática docente na área da saúde: conceitos, paradigmas e inovações. Washington: OPAS; 2005.

16. Tavares V. O ambiente inovador da EAD como agente das mudanças e transformações das práticas pedagógicas. 2007 [Acesso em 26 jan. 2007]; Disponível em: http://www.elearningbrasil.com.br/home/artigos/artigos.asp?id=3886.

17. Bastos LF, Macedo MM. Novas fronteiras da educação: educação à distância Rev bras odontol 2002 mar.-abr.;59(2):112-5.

18. Nascimento FP, Carnielli BL. Educação a distância no ensino superior: expansão com qualidade? ETD - Educação Temática Digital 2007 9(1):84-98.

REV, ODONTOL.

UNIV, CID, SÃO

PAULO

2012; 24(1): 6-14, JAN-ABR 
DOTTA EAV

GARCIA PPNS

CANDIDO LM

ELABORAÇÃO

DE UM CURSO

INTERATIVO

VOLTADO AO

APRENDIZADO

DE UM SISTEMA

APLICATIVO EM

ODONTOLOGIA

UTILIZANDO

A PLATAFORMA

MOODLE

14

19. Moran J. Mudar a forma de ensinar e de aprender com tecnologias. Interações estud pesqui psicol 2000 jan.-jun.;5(9):57-72.

20. Santos ML. A futura universidade e os cursos sequenciais Folha méd 2000 jul.-set.;119(3):7.

21. Alves L, Brito M. O ambiente moodle como apoio ao ensino presencial. 2009 [Acesso em 22 out. 2009]; Disponível em: http://www.abed.org.br/congresso2005/ por/pdf/085tcc3.pdf

22. Valente J. Mudanças na sociedade, mudanças na educação: o fazer e o compreender. In: Valente J. O computador na sociedade do conhecimento. Campinas: Nied/ Unicamp; 1999.

23. Moran JM. Modelos e avaliação do ensino superior a distância no Brasil. ETD - Educação Temática Digital 2009 10(2):54-70.

24. Lopes A, Gomes $M$, editors. Ambientes virtuais de aprendizagem no contexto do ensino presencial: uma abordagem ref exiva. Challenges 2007: Actas da V Conferência Internacional de TIC na Educação Braga: Centro de Competência da Universidade do Minho; 2007.

25. Sistemas de Formação Multimédia. A plataforma Moodle. 2010 [Acesso em 01 mar. 2010]; Disponível em: http://www.sfm.pt/e-learning/lms-moodle.php.

26. Dotta EAV, Teles GH. Sistemas aplicativos para uso odontológico RGO (Porto Alegre) 2003 abr.-jun.;51(2):119-22.

27. Dotta E. Conhecimento e utilização da informática pelo cirurgião-dentista como ferramenta de trabalho [Relatório trienal referente ao período de 2001 a 2003]. Araraquara: Faculdade de Odontologia da UNESP; 2004.

28. Azevedo T, Bittencourt R. Ref exões sobre o planejamento pedagógico de um curso de extensão universitária na modalidade a distância. 2005 [Acesso em 17 nov. 2005]; Disponível em: www.abed.org.br.

29. Bernardino S, Fernandes N. Manual de utilização da plataforma Moodle. 2009 [Acesso em 28 out. 2009]; Disponível em: http://www.antonioarroio.crie.fc.ul.pt/ f le.php/1/Manuais_de_Utilizacao_Professores/Manual_do_moodle.pdf.

30. Monteiro M. A informática no processo ensino aprendizagem. 2009 [Acesso em 26 nov. 2009]; Disponível em: http://www.concemorais.blogspot. com/2009/05informatica-do-processo-ensino.html.

31. Coscarelli C. O uso da informática como instrumento de ensino-aprendizagem. Presença Pedagógica 1998 mar./abr.;4(20):37-45.

32. Bottentuit JR J, Coutinho C, editors. Uso da plataforma de ensino a distância WebCT numa disciplina de mestrado. Congresso da Sociedade Portuguesa de Ciência da Educação (IX SPCE); 2007; Funchal, Portugal.

33. Gonçalves J, Salvador J. Educação a distância e o Moodle: novas formas de ensinar e de aprender matemática. 2009 [Acesso em 23 out. 2009]; Disponível em: http:// www.sbem.com.br/f les/IX-enen/Html/minicursos.html.

Recebido em 13/07/2011

Aceito em: 15/09/2011

REV. ODONTOL.

UNIV. CID. SÃO:

PAULO 